\title{
Resolved Scalar Mixing in Large Eddy Simulations of a Low Reynolds Number Plane Mixing Layer
}

\author{
S. N. Hug* and W. A. McMullan ${ }^{\dagger}$ \\ Dept. Engineering, University of Leicester, LE1 7RH, United Kingdom
}

\begin{abstract}
In this paper we present Large Eddy Simulations of a low Reynolds number plane mixing layer. The purpose of the research is to assess the effect of inflow conditions on the scalar mixing in the flow. A high-resolution grid is used to study a pre-transition mixing layer originating from initially laminar conditions. Both pseudo-random white noise and physically-correlated fluctuations produced by an inflow generation technique provide the background fluctuations for the simulations. It is found that the scalar statistics are largely spanwise invariant when pseudo-random fluctuations are employed, whilst large spanwise variations in the scalar statistics are observed when physically-correlated fluctuations provide the background disturbances. The large fluctuations are attributed to the presence of organised streamwise vortices in the mixing layer. The implications of this research in terms of the documentation of experimental initial conditions is discussed.
\end{abstract}

\section{Introduction}

The mixing of fluid is an important natural process, and has many applications in modern engineering. In particular, the mixing of chemical species to produce reaction products, including exothermic reactions in combustion systems, is extremely relevant to current demands of increasing energy efficiency and reduction of pollutant formation. A comprehensive understanding of mixing processes in fluids flows is therefore essential.

The plane mixing layer is geometrically the simplest form of free shear flow. In this configuration two parallel streams of fluid of differing velocity (and possibly density) merge downstream of a splitter plate. Classical turbulence theory suggests that the plane turbulent mixing layer is an essentially random flow field, but experiments have observed organised vortex structures in the flow over the entire range of Reynolds numbers studied. ${ }^{1-8}$ These structures occupy the entire visual thickness of the flow and are therefore dynamically significant. At low Reynolds number, the mixing layer evolves through successive pairings of the primary Kelvin-Helmholtz $(\mathrm{K}-\mathrm{H})$ vortices, with a step-wise growth of the flow resulting from the interaction. ${ }^{1}$ At higher Reynolds numbers, however, continuous linear growth appears to be the dominant growth mechanism. ${ }^{3}$ The distinction between the regions of step-wise and continuous linear growth is marked by the mixing transition, ${ }^{7}$ where the flow undergoes the transition to turbulence. The transition is triggered by a pairing interaction between primary vortices, following the formation of secondary streamwise vortices. ${ }^{9}$ The transition normally occurs once the local Reynolds number, based on the visual thickness of the layer and the velocity difference across it, exceeds $10,000 .{ }^{10}$

Research into the mixing processes in the shear layer are important as the entrainment and mixing of fluid in the flow is largely controlled by large scale structure dynamics. Experimental research into the molecular mixing of fluid in the mixing layer has been performed using both passive scalar and chemical reaction techniques. In the passive scalar method, a tracer is seeded into one of the freestreams, and its concentration in the mixing layer is recorded. The mixing of the scalar is commonly represented by the probability density function (p.d.f.) of the passive scalar. Experimental measurements have produced three differing types of p.d.f. distributions. The first is the marching p.d.f., where the most probable scalar concentration at a point in the mixing layer is equal to the average scalar at that point. The second is the non-marching p.d.f., where the most probable scalar concentration has a constant value across the mixing layer. The final type is the

\footnotetext{
* Research Student.

${ }^{\dagger}$ Lecturer, AIAA Senior Member.
} 
tilted p.d.f., where the preferred scalar concentration varies somewhat across the mixing layer. This tilted p.d.f. is commonly viewed as a hybrid between the marching and non-marching p.d.f.s.

Scalar concentration measurements of the gaseous pre-transition mixing layer revealed a non-marching p.d.f. distribution. ${ }^{11,12}$ Beyond the mixing transition, both aqueous ${ }^{12}$ and gaseous ${ }^{7}$ experiments have produced non-marching p.d.f.s., with an entrainment bias evident towards the high-speed stream. Other experiments, however, have shown that both tilted, ${ }^{4,13}$ and marching p.d.f.s can be obtained. ${ }^{14}$ These studies suggest that the obtained p.d.f. may well be a function of the local Reynolds number, or the local value of the pairing parameter. ${ }^{4}$ Further scalar p.d.f. measurements have found that the shape of the p.d.f. may well depend on the initial conditions of the mixing layer. ${ }^{16}$ Reacting flow experiments have demonstrated that gaseous mixing layers contain twice as much fluid mixed at the molecular level when compared with aqueous flows. ${ }^{17}$ So-called 'flip' experiments, where chemical reactants are interchanged between the freestreams, have confirmed the asymmetric entrainment bias of the mixing layer, with an excess of fluid entrained from the high-speed stream. ${ }^{15}$

Numerical simulation should permit careful measurement of the scalar p.d.f., and offer an environment in which the effect of the initial conditions on the scalar mixing can be assessed. Direct Numerical Simulation (DNS) studies of the temporal mixing layer demonstrated that the character of the scalar p.d.f. depends on the initial conditions of the flow. ${ }^{18,19} \mathrm{~A}$ temporal mixing layer dominated by $\mathrm{K}-\mathrm{H}$ type vortices produces a non-marching p.d.f., whilst marching p.d.f.s are obtained when the flow is subjected to higher turbulence levels. ${ }^{18}$ A Direct Numerical Simulation (DNS) study of the spatially-developing flow has produced evidence of marching p.d.f. behaviour in the idealised mixing layer, ${ }^{20}$ for only one local Reynolds number. Mixing layers are commonly used as validation test cases for new numerical methods, and the majority of other numerical simulations including p.d.f. measurements in the archival literature are of mixing layers confined to two-dimensional boxes. ${ }^{21,22}$ Whilst these simulations are attractive on grounds of computational cost, it has been shown that mixing layers confined to two-dimensional domains do not undergo the mixing transition, as the formation of the secondary instability is inhibited. ${ }^{23,24}$ This has a knock-on effect on the growth of the mixing layer, and entrainment of fluid into it. ${ }^{23}$ Furthermore, the specification of idealised inflow conditions with white noise disturbances, common in mixing layer simulations, ${ }^{20,25}$ can lead to different types of streamwise vorticity being formed in the mixing layer when compared to highly two-dimensional white noise fluctuations ${ }^{26}$ or physically-correlated inflow disturbances. ${ }^{27}$

In this study high resolution Large Eddy Simulations of the pre-transition mixing layer are performed. The simulations are based on the experimental data of Masutani \& Bowman, ${ }^{11}$ which were confined to the pre-transition region of the mixing layer. In the experiment, velocity fluctuation profiles were not specified for each stream and as such the initial conditions are underspecified for replication by numerical simulation. The effect of the inflow conditions on scalar mixing is assessed through the variation of both the nature and the magnitude of the imposed fluctuations. White-noise perturbations that are commonly imposed in numerical simulations ${ }^{20,28}$ provide one fluctuation environment, whilst an inflow generation technique is used to provide physically correlated fluctuations in the other simulations. As this experiment considered a low Reynolds number mixing layer,we primarily focus on the resolved scalar mixing on the high-resolution grid employed here. The effects of using a stochastic probability density function method to account for subgrid-scale mixing is beyond the scope of the present work.

\section{Numerical Methods}

The spatially filtered equations for conservation of momentum and mass for a uniform density fluid are

$$
\begin{gathered}
\frac{\partial \bar{u}_{i}}{\partial t}=-\frac{\partial \bar{p}}{\partial x_{i}}+\frac{\partial}{\partial x_{j}}\left(-\bar{u}_{i} \bar{u}_{j}+2 \nu \bar{S}_{i j}\right) \\
\bar{S}_{i j}=\frac{1}{2}\left(\frac{\partial \bar{u}_{i}}{\partial x_{j}}+\frac{\partial \bar{u}_{j}}{\partial x_{i}}\right) \\
\frac{\partial \bar{u}_{i}}{\partial x_{i}}=0
\end{gathered}
$$

These equations are discretised on a staggered mesh. The viscosity $\nu$ can consist of both a molecular and a subgrid component, $\nu=\nu_{m}+\nu_{s g}$, if a subgrid-scale model is used. In this study the Wale Adapting Local Eddy-viscosity (WALE) model ${ }^{29}$ is utilised, with the eddy viscosity, $\nu_{s g}$, calculated by 


$$
\nu_{s g}=\left(C_{w} \Delta\right)^{2} \frac{\left(S_{i j}^{d} S_{i j}^{d}\right)^{3 / 2}}{\left(\bar{S}_{i j} \bar{S}_{i j}\right)^{5 / 2}+\left(S_{i j}^{d} S_{i j}^{d}\right)^{5 / 4}}
$$

where $S_{i j}^{d}=\frac{1}{2}\left(\bar{g}_{i j}^{2}+\bar{g}_{j i}^{2}\right)-\frac{1}{3} \delta_{i j} \bar{g}_{k k}^{2}, \bar{g}_{i j}=\partial \bar{u} / \partial x$, and $C_{w}$ is a model constant specified a priori. The WALE model is attractive for the simulation of free shear flows with initially laminar conditions, as it predicts zero eddy viscosity in the presence of pure shear. It has been shown in other work by the author that this model produces improved plane mixing layer predictions when compared to the standard Smagorinsky model. ${ }^{28}$

Temporal advancement of the governing equations is performed by the Adams-Bashforth method. In this method, a provisional velocity field is obtained through

$$
\bar{u}_{i}^{*}=\bar{u}_{i}^{n}+\Delta t\left(\frac{3}{2} H_{i}^{n}-\frac{1}{2} H_{i}^{n-1}\right)
$$

with

$$
H_{i}=\frac{\partial}{\partial x_{j}}\left(-\bar{u}_{i} \bar{u}_{j}+2 \nu \bar{S}_{i j}\right)
$$

The provisional velocity $\bar{u}_{i}^{*}$ does not obey continuity, and is updated to the actual velocity at the next time step, $\bar{u}_{i}^{n+1}$, by the pressure solver. The pressure field is solved implicitly by the use of the continuity equation. The provisional velocity field of $\bar{u}_{i}^{*}$ is used to derive the actual velocity by including the gradient of an unknown pressure field $\bar{p}^{n+\frac{1}{2}}$, such that

$$
\bar{u}_{i}^{n+1}=\bar{u}_{i}^{*}-\Delta t \frac{\partial \bar{p}^{n+\frac{1}{2}}}{\partial x_{i}}
$$

As the new velocity field must have zero divergence, a Poisson equation can be found for the pressure field between the present and next time step

$$
\nabla^{2} \bar{p}^{n+\frac{1}{2}}=\frac{1}{\Delta t} \frac{\partial \bar{u}_{i}^{*}}{\partial x_{i}} \equiv R
$$

This equation is then solved using a multi-grid method to increase the speed of convergence of the solution. A standard convective outflow condition is applied at the outflow plane of the computational domain.

For the purposes of flow visualisation a passive scalar is also introduced into the flow domain, which is governed by the equation

$$
\frac{\partial \bar{\xi}}{\partial t}=\frac{\partial}{\partial x_{i}}\left(-\bar{u}_{i} \bar{\xi}+\alpha \frac{\partial \bar{\xi}}{\partial x_{i}}\right)
$$

where $\alpha$ is the diffusivity, which contains both a molecular and a subgrid component, $\alpha=\alpha_{m}+\alpha_{s g}$, if a subgrid-scale model is used. With a subgrid scale model employed, the subgrid diffusivity is set to $\alpha_{s g}=\nu_{s g} / 0.3$. The scalar is discretised on the staggered mesh at the cell centre, and second-order upwinding scheme is used to calculate the scalar flux between cell faces. The Adams-Bashforth method is used to integrate the scalar field forward in time, with a method of discretisation very similar to equations 5-6.

The simulations requiring a physically correlated inflow fluctuations obtain these from the recycling and rescaling method of Xiao.et al. ${ }^{30}$ This method requires virtual domains to be placed upstream of the main simulation domain, in which the correlated disturbances are generated. The flow in the virtual domain then provides the inflow condition for the main simulation domain. This method has been used extensively in recent research, being used to specify inflow conditions for plane mixing layers, ${ }^{30,31}$ axisymmetric jet flows, ${ }^{32}$ and multiphase flow. ${ }^{33}$ In all of these flow configurations, the turbulent inlet condition produced main simulation flow-fields with accurate flow statistics.

\begin{tabular}{ccccc}
$U_{1}\left(\mathrm{~ms}^{-1}\right)$ & $\theta_{1}(\mathrm{~mm})$ & $U_{2}\left(\mathrm{~ms}^{-1}\right)$ & $\theta_{2}(\mathrm{~mm})$ & $\mathrm{R}$ \\
\hline 6.0 & 0.24 & 3.0 & 0.28 & 0.333 \\
\hline
\end{tabular}

Table 1. Flow parameters. 


\section{Simulation Set-up}

The experiments conducted by Masutani \& Bowman provide the reference data for the present simulations. ${ }^{11}$ In these experiments a small, low-speed experimental facility was used to investigate scalar mixing in the pre-transition mixing layer. The test section of the wind tunnel had an adjustable lower wall to minimise the streamwise pressure gradient in the flow. The boundary layers at the splitter plate trailing edge were laminar in nature, with the freestream turbulence intensity being less than $0.5 \% U$, where $U$ is the freestream. No velocity fluctuation statistics in the boundary layers were reported, however. The bulk flow parameters are outlined in Table 1 . The velocity ratio parameter of the flow, $R$, is defined as

$$
R=\frac{U_{1}-U_{2}}{U_{1}+U_{2}}
$$

where $U_{1}$ and $U_{2}$ are the high- and low-speed stream velocities respectively. In the experiment, $\mathrm{O}_{3}$ with concentration 1000 p.p.m. was seeded in the low-speed stream and this was assumed to behave as a passive scalar. Mean statistics and probability density functions were obtained for this scalar.

\section{A. Computational Parameters}

The conditions outlined in Table 1, along with a description of the freestream turbulence intensity, defines the initial conditions of the experiment. As was noted in the introduction this information does not represent a complete description of the mixing layer initial conditions and as such any attempt to simulate this flow will be, at best, an approximation of the experimental conditions. Given that no information is available pertaining to the velocity fluctuation statistics in the laminar separating boundary layers, this paper attempts to study the influence of different fluctuation profiles imposed onto the base laminar inflow. Furthermore, the influence of the nature of the imposed fluctuations will be assessed. Both white noise disturbances, and physically-correlated disturbances provided by an inflow generation method will be employed.

Four distinct simulations are performed here; two simulations have white noise disturbances ('WN'), and two have physically-correlated disturbances ('RRM'). For each type of imposed fluctuation, a low-level disturbance (' $\mathrm{L}$ ') and high-level disturbance ('H') environment is imposed on the high-speed side laminar boundary layer. The mean streamwise velocity and associated fluctuation profiles in the high-speed stream of these simulations are shown in Figure 1. Similar profiles are imposed in the low-speed stream. The simulation inflow parameters are summarised in Table 2.

The outflow condition is a standard advective condition that ensures global mass conservation in the computational domain. The upper and lower guidewalls of the domain are modelled as free-slip boundaries. The WALE subgrid scale model has its model coefficient set to $C_{w}=0.56$. A previous study has shown that the evolution of the mixing layer is insensitive to a range of WALE model coefficient on a grid of similar resolution to that used here. ${ }^{28}$ The passive scalar is given a value of unity in the low-speed stream, and zero in the high-speed stream, in the same manner as the experiment. The simulations have a time step of $\Delta t=5 \times 10^{-6} \mathrm{~s}$, which produces a CFL number of less than 0.35 throughout the computation. Upon the flow field attaining a statistically stationary state, flow statistics are obtained over a period corresponding to 18 convective flow-through times, based on the mean convection velocity of the flow $U_{c}=0.5\left(U_{1}+U_{2}\right)$. Velocity statistics are spanwise-averaged to increase the total number of samples. Cross-plane $y-z$ measurement of the flow are recorded at $x=0.07,0.11$, and $0.15 \mathrm{~m}$, with 1500 samples providing data for ensemble averaged scalar statistics and probability density functions across the entire span of the domain.

Simulation setup details specific to each inflow generation technique are described below.

\section{Idealised Simulation}

For the simulations where white noise fluctuations are imposed on a mean inflow velocity profile, the computational domain comprises the region downstream of the trailing edge of the splitter plate. No solid geometry is included in the simulation domain. This domain extends $0.2 \times 0.04 \times 0.1(\mathrm{~m})$ in the streamwise, crossstream, and spanwise directions respectively at the inflow plane. The lower guidewall of the domain is angled in order to minimise the streamwise pressure gradient in the flow, as was the case in the experiments. The computational domain can therefore be considered as a reasonably accurate representation of the experimental test section. The domain is discretised into $512 \times 256 \times 152$ cells. The grid is refined in the plane of the splitter plate, with a minimum grid spacing of $\Delta x_{\min }=1.1 \times 10^{-4} \mathrm{~m}, \Delta y_{\min }=9 \times 10^{-5} \mathrm{~m}$. Grid stretching is 
performed in both directions to maximise the number of cells in regions of steep velocity gradients. This grid resolution is similar to that of previous LES mixing layer studies, ${ }^{28}$ where good results were obtained. The maximum ratio of subgrid-to-kinematic viscosity recorded in all simulations was $\nu_{s g} / \nu_{m} \approx 3$, demonstrating that the mesh has a high level of resolution for this particular flow configuration.

This type of simulation, with a Blasius inflow velocity profile and white noise fluctuations, can be considered as an idealised form of the experimental conditions.

\section{Correlated Inflow Simulations}

The inflow generation technique requires virtual domains in which the physically correlated fluctuations are generated. In both freestreams, the virtual domain stretches $0.026 \mathrm{~m}$ upstream of the trailing edge of the splitter plate. In these virtual domains, the splitter plate is modelled as a solid boundary of infinitesimal thickness. The grid spacing in the virtual domains matches that found in the initial region of the mixing layer domain. Each virtual domain contains $256 \times 128 \times 152$ cells. The simulations described above are repeated with the inflow generator providing physically-correlated fluctuations, with the disturbance profiles consistent with those of the WN cases. Aside from the extra computational domain and inlet generation method, all other simulation parameters remain constant with respect to the idealised simulation described above.

\begin{tabular}{ccc} 
Case & High-speed fluctuations & Low-speed fluctuations \\
\hline WNL & Fig. 1b & Fig. 1b \\
WNH & Fig. 1c & Fig. 1b \\
RRML & Fig. 1b & Fig. 1b \\
RRMH & Fig. 1c & Fig. 1b \\
\hline
\end{tabular}

Table 2. Simulation inflow parameters. Figure references correspond to the fluctuation profiles used in the respective stream.

\section{Results}

The simulation results are normalised in the same manner as the experiments. For the velocity field, the statistics are transformed into a non-dimensional co-ordinate system, $\eta=\left(y-y_{0}\right) /\left(x-x_{0}\right)$, where $y_{0}$ is the cross stream location at which the flow velocity is equal to the convection velocity, $U_{c}$, and $x_{0}$ is the virtual origin. Concentration statistics are normalised into the co-ordinate system, $\eta^{\prime}=\left(y-y_{0}^{\prime}\right) /\left(x-x_{0}^{\prime}\right)$, where $x_{0}^{\prime}, y_{0}^{\prime}$ are the concentration analogues to $x_{0}$ and $y_{0}$.

\section{A. Velocity Statistics}

Mean streamwise velocity profiles are shown in Figure 2a-2b for Cases WNL and RRMH. In Case WNL the profile at $x=0.12 \mathrm{~m}$ shows some deviation from the experimental data. Further downstream, however, the predictions agree much better with the experiment. Case RRMH (along with WNH and RRML, not shown here) produce good agreement with the experimental data. All cases show some discrepancies in the predicted streamwise velocity fluctuations, $u_{r . m . s .}$, however. The experiment predicts a reasonably high fluctuation level in the freestreams $(\sim 5 \%)$, which is significantly higher than the freestream turbulence intensity measured at the splitter plate trailing edge. This may be caused by the influence of the guidewall boundary layers on the freestream flow, given the narrow vertical extent of the test section. In addition, the velocity statistics presented from the simulations have been spanwise averaged, whereas the experimental data was recorded at a single spanwise plane. Neither the experiment nor the simulations presented here produce velocity fluctuations which collapse onto a single self-similar profile, and as such the mixing layer does not attain self-similarity in the streamwise extent considered here. 


\section{B. Flow Visualisation}

A common method to visualise a flow-field in experiments is through Planar Laser Induced Fluorescence (PLIF). A numerical analogue of this technique is to interrogate planes of the instantaneous passive scalar distribution in the flow. Typical 'side-view' single-plane scalar images are shown in Figures 4 and 5 for Cases WNL and RRML respectively. These images share some common features; there is an extended vortex sheet immediately downstream of the splitter plate trailing edge, from which vortex structure emerge. The vortex structure becomes somewhat irregular further downstream in the domain, which implies that some form of three-dimensionality is present within the flow. These low Reynolds number mixing layers do not, however, undergo the transition to turbulence within the computational domain.

The underlying three-dimensionality of the mixing layer can be qualitatively investigated through inspection of cross-plane $(y-z)$ images of the passive scalar. Sample instantaneous images, recorded at $x=0.07$, 0.11, and 0.15m, are shown in Figures 6-9 for Cases WNL, WNH, RRML, and RRMH respectively. These images are recorded when an interconnecting braid region between primary vortices is passing through the sampling plane. At the most upstream station in Case WNL (Figure 6a), a long-wavelength secondary instability co-exists with the primary structure. This instability has the appearance of an undulation in the scalar distribution across the span of the mixing layer. This type of secondary structure has been observed in other mixing layer simulation originating from initially-laminar conditions with a white noise disturbance environment. ${ }^{27,34}$ Further downstream (Figures 6b-6c) a single row of 'mushroom-shaped' eruptions is visible along the span of the braid region, indicating the presence of a row of streamwise vortices. In Case WNH (Figure 7) a similar cross-plane scalar evolution is observed, although the wavelength of the secondary instability at $x=0.07 \mathrm{~m}$ is qualitatively much shorter than that of Case WNL.

The scalar distribution in Case RRML reveals an interesting difference caused by the correlated inflow fluctuations. At $x=0.07 \mathrm{~m}$ (Figure 8a), a short-wavelength spanwise variation in the scalar distribution is visible across the span. This particular image captures the braid region just as the next vortex core passes through the sampling plane, which results in the presence of a second row of structures appearing on the upper side of the mixing layer. As the flow evolves downstream the scale of the secondary structure increases in conjunction with the local visual thickness of the mixing layer. Qualitatively these images are very similar to those obtained in experiments of low Reynolds number mixing layers. ${ }^{6}$ In Case RRMH (Figure 9) the short-wavelength instability does not appear to be present at $x=0.07 \mathrm{~m}$ (Figure 9a), but a secondary structure has emerged at $x=0.11 \mathrm{~m}$ in Figure $9 \mathrm{~b}$.

The cross-stream $(y-z)$ flow visualisations presented here show, in an instantaneous sense, some significant variations in the passive scalar distribution across the span of the mixing layer. Of course, caution must be exercised when interpreting individual instantaneous realisations of the flow. A more quantitative statistical description of the cross-plane flow fields is required in order to further analyse the influence of the inflow conditions on the simulated mixing layers. This statistical analysis is described below.

\section{Spanwise Dependence of Mixing Layer Properties}

Sequences of up to 1,500 cross-stream flow visualisations are used to produce time series of data at $x=0.07$, 0.11 , and $0.15 \mathrm{~m}$ across the entire spanwise domain, with results from $x=0.11 \mathrm{~m}$ presented here. From these sequences, ensemble averaged velocity statistics, scalar statistics, and probability density functions are computed. That the entire $y-z$ flow field is sampled at each streamwise location permits the study of the spanwise variation of the flow statistics.

In Figure 10 the mean scalar profiles from the simulations are presented. Included in each image is the spanwise-averaged scalar field, along with two profiles chosen randomly from across the span. Both white noise disturbance simulations (Figures 10a-10b) show a high-level of spanwise uniformity in the scalar profile, in that the spanwise-averaged profile closely follows those recorded at individual spanwise planes. The mean scalar profile of Case WNL produces reasonably good agreement with the experimental data. In contrast, both correlated fluctuation inflow simulations show significant variations in the scalar profiles across the span. The single-plane profiles deviate substantially from the spanwise-averaged data, implying that the mixing layer is three-dimensional at this streamwise location. Whilst the spanwise-averaged data from both Cases RRML and RRMH show poor agreement with the experimental data in the region of $0.05 \leq \eta^{\prime} \leq 0.1$, certain single plane profiles do provide much better agreement. In the same manner, other single-plane profiles show much larger deviations from the experimental data.

Similar trends are observed in the scalar fluctuation profiles shown in Figure 11. None of the simulations 
predict particularly good agreement with the experimental data at this location, a feature that is common with other numerical simulations of this reference dataset. ${ }^{21,22}$ As with the mean scalar profile, the white noise fluctuation simulations show no significant spanwise variations in the scalar fluctuation, whilst the correlated inflow simulations produce widely different profiles dependent on spanwise location.

The probability density function computed here is defined in the usual manner. ${ }^{4,12}$ The p.d.f. of $\bar{\xi}$ at a point $y$ in the layer is denoted as $p(\bar{\xi}, y)$. The p.d.f. is normalised such that

$$
\int_{0}^{1} p(\bar{\xi}, y) d \bar{\xi}=1
$$

and the entrainment ratio of the mixing layer is computed directly from the probability density function. ${ }^{4}$ In the reference experiment, a non-marching p.d.f. with a preferred scalar concentration of $\xi=0.2$ was recorded at $x=0.11 \mathrm{~m}$. Sample p.d.f.s from Cases WNL at this streamwise location are shown in Figure 12 . At both spanwise positions a non-marching p.d.f. is predicted, although the preferred concentration of $\bar{\xi} \approx$ 0.34 is significantly higher than that reported in the experiment. The p.d.f.s obtained elsewhere across the span do not change substantially from those shown here, implying that the flow evolution in Case WNL is largely two-dimensional. Qualitatively similar p.d.f.s are obtained in Case WNH.

The p.d.f.s recorded at $x=0.11 \mathrm{~m}$ in Case RRML, presented in Figure 13, do have markedly different shapes across the span of the mixing layer. At $z=0.061 \mathrm{~m}$ (Figure 13a), the p.d.f. shows a preferred concentration of $\bar{\xi} \approx 0.17$ on the upper side of the mixing layer. This preferred concentration is close to the experimentally reported value. The preferred concentration on the low-speed side does, however, does change with vertical position in the mixing layer. At $x=0.0967 \mathrm{~m}$ (Figure 13b), a clear non-marching p.d.f. is present, with a preferred concentration of $\bar{\xi} \approx 0.3$. Again, this preferred concentration is significantly higher than that reported in the experiment. The large differences in the computed p.d.f.s is repeated at all other measurement stations, and is also observed in Case RRMH. These results imply that the statistical properties of the mixing layers which originate from laminar boundary layers with correlated fluctuations are heavily dependent on the spanwise location at which the measurements are taken.

Changes in the shape of the probability density function will necessarily produce variations in the entrainment ratio of the mixing layer. The entrainment ratio is computed directly from the p.d.f. following the method of Karasso and Mungal. ${ }^{4}$ For each simulation considered here, the entrainment ratio is plotted across the span at $x=0.11 \mathrm{~m}$ in Figure 14. Also included on these plots is the locus of the centreline of the mixing layer, $y_{0}$, which is defined as the vertical location where the mean velocity equals the convection velocity of the flow, $U_{c}$. It has been shown both experimentally and numerically that the centreline locus can be used to infer the presence of stationary streamwise vorticity in the mixing layer. ${ }^{35,36}$ Cases WNL and WNH (Figures 14a and 14b) show no particular periodicity in the variation of the mixing layer centreline across the span, and any variation across the span has a low amplitude. In the simulations where correlated fluctuations provide the inflow disturbances, however, there is evidence for significant spanwise variations in both the mixing layer centreline and the entrainment ratio. The local entrainment ratio can vary by as much as 30\% across the span of the domain in both simulations. It is interesting to note that the peaks and troughs in the entrainment ratio profile are anti-correlated with the peaks and troughs in the mixing layer centreline. Experiments and numerical simulations have noted that the maxima and minima in the centreline distribution can be associated with the presence of statistically stationary streamwise vorticity, ${ }^{35,36}$ and this is explored further here.

Contours of secondary shear stress, $\overline{u^{\prime} w^{\prime}}$, normalised by the square of the velocity difference across the mixing layer, $U_{0}=\left(U_{1}-U_{2}\right)$, are presented at $x=0.11 \mathrm{~m}$ for in each simulation in Figures 15 . The secondary shear stress contours of Cases WNL and WNH in Figures 15a-15b are very similar, in that there is no particular clustering of $\overline{u^{\prime} w^{\prime}}$ across the span. This irregular distribution of $\overline{u^{\prime} w^{\prime}}$ has been reported in other simulations of high Reynolds number turbulent mixing layers, ${ }^{27}$ and indicates that any streamwise vorticity present in the mixing layer originating from initially laminar conditions with a white noise disturbance environment is not statistically stationary. In Figure $15 \mathrm{c}$, the $\overline{u^{\prime} w^{\prime}}$ distribution of Case RRML shows evidence of clustering of the secondary shear stress. There is some alternate-sign banding of $\overline{u^{\prime} w^{\prime}}$ across the span of the mixing layer, which indicates the presence of organised streamwise vorticity in the flow. The character of this streamwise vorticity is quite chaotic however, and the flow has not attained a single row of streamwise vortices that has been observed in both experiments and other numerical simulations. ${ }^{35,36}$ At this streamwise measurement station the pairing parameter, $x_{i}^{*}=R x /\left(30 \theta_{i}\right)$ has a value of $x_{i}^{*}=5.05$. In other experiments, the secondary shear stress contours had a similar distribution for a comparable pairing parameter value. ${ }^{35}$ 
The secondary shear stress contours at $x=0.11 \mathrm{~m}$ in Case RRMH (Figure 15d) are qualitatively similar to those of Case RRML, in that there are clusters of shear stress embedded across the span of the mixing layer. The precise details of the secondary shear stress distribution are, however, substantially different to that of Case RRML. Given that the local pairing parameter has the same value at this streamwise station in both simulations, it is clear that the magnitude of the imposed physically-correlated laminar boundary layer disturbances has a substantial impact on the development of the streamwise vorticity in the mixing layer. The sensitivity of the streamwise vortex structure to small changes in the initial conditions has been noted in past experiments. ${ }^{6,37}$

\section{Summary}

The simulations presented here show that the scalar mixing in the pre-transition mixing layer is sensitive to initial conditions. Simulations which originate from initially-laminar conditions with a white noise disturbance environment are largely statistically two-dimensional, in that the computed scalar statistics do not vary significantly across the span. For initially-laminar simulations with physically-correlated inflow conditions the scalar statistics are found to have a large spanwise variation. The entrainment ratio and probability density function of the scalar are heavily dependent on spanwise measurement location, and it is plausible that widely different estimations for these quantities can be obtained from pure single-spanwiseplane analysis of the simulation. As all experiments have physically-correlated background disturbances, great care must be taken to document the magnitude of these fluctuations, as they may well have a large impact on the measured flow statistics. Single-plane experimental analysis may also be inappropriate for mixing layers containing an organised secondary streamwise structure.

\section{References}

\footnotetext{
${ }^{1}$ Winant C.D., and Browand F.K., "Vortex Pairing: The mechanism of turbulent mixing layer growth at moderate Reynolds numbers," Journal of Fluid Mechanics, Vol 63, 1974 pp. 237-255.

${ }^{2}$ Brown G.L., and Roshko A., "On density effects and large structure in turbulent mixing layers," Journal of Fluid Mechanics, Vol. 64, 1974, pp. 755-816.

${ }^{3}$ D'Ovidio, A., and Coats, C.M., "Coherent-structure evolution in turbulent mixing layers. Part 1: Experimental evidence," Journal of Fluid Mechanics, Vol. 737, 2013, pp. 466-498.

${ }^{4}$ Karasso P.S., and Mungal M.G., "Scalar mixing and reaction in plane liquid shear layers," Journal of Fluid Mechanics, Vol. 323, 1996, pp. 23-63.

${ }^{5}$ Slessor, M.D., Bond, C.L., and Dimotakis, P.E. "Turbulent shear-layer mixing at high Reynolds numbers: effects of inflow conditions," Journal of Fluid Mechanics, Vol. 375, 1998, pp. 115-138.

${ }^{6}$ Bernal L.P., and Roshko A., "Streamwise vortex structures in plane mixing layers," Journal of Fluid Mechanics, Vol. 170, 1986, pp. 499-525.

${ }^{7}$ Konrad J.H., "An experimental investigation of mixing in two-dimensional shear flows with applications to diffusion limited chemical reactions," PhD thesis, California Institute of Technology, 1976.

${ }^{8}$ Dimotakis P.E., and Brown G.L., "The mixing layer at high Reynolds number: large scale structure and entrainment," Journal of Fluid Mechanics, Vol. 78, 1976, pp. 535-560.

${ }^{9}$ Huang L-S, and Ho C-M., "Small scale transition in a plane mixing layer", Journal of Fluid Mechanics, Vol 210, 1990, pp. $475-500$.

${ }^{10}$ Dimotakis P.E., "The mixing transition in turbulent flows," Journal of Fluid Mechanics, Vol. 409, 2000, pp. 69-98.

${ }^{11}$ Masutani, S.M, and Bowman, C.T., "The structure of a chemically reacting plane mixing layer," Journal of Fluid Mechanics, Vol. 172, 19786, pp. 93-126.

${ }^{12}$ Koochesfahani M.M., and Dimotakis P.E., "Mixing and chemical reactions in a turbulent liquid mixing layer," Journal of Fluid Mechanics, Vol. 170, 1986, pp. 83-112.

${ }^{13}$ Meyer, T.R., Dutton, J.C., and Lucht, R.P., "Coherent structures and turbulent molecular mixing in gaseous planar shear layers," Journal of Fluid Mechanics, Vol. 558, 2006, pp. 179-205.

${ }^{14}$ Batt, R.G., "Turbulent mixing of passive and chemically reacting species in a low-speed shear layer," Journal of Fluid Mechanics, Vol. 82, 1986, pp. 53-95.

${ }^{15}$ Koochesfahani, M.M., Dimotakis, P.E., and Broadwell, J.E., "A "flip" experiment in a chemically reacting turbulent mixing layer," AIAA Journal, Vol. 23, 1985, pp. 1191-1194.

${ }^{16}$ Picket, L.M., and Ghandhi, J.B., "A Passive scalar mixing in a planar shear layer with laminar and turbulent initial conditions," Physics of Fluids, Vol. 14, 2002, pp. 985-998.

${ }^{17}$ Breidenthal, R., "Structure in turbulent mixing layers and wakes using a chemical reaction," Journal of Fluid Mechanics, Vol. 109, 1981, pp. 1-24.

${ }^{18}$ Rogers M.M.,and Moser R.D., "Direct simulations of a self-similar turbulent mixing layer," Physics of Fluids, Vol. 6, 1994, pp. 903-923.
} 
${ }^{19}$ Ansari, A., "Self-similarity and mixing characteristics of turbulent mixing layers starting from initially laminar conditions," Physics of Fluids, Vol. 9, 1997, pp. 1714-1728.

${ }^{20}$ Attili, A., and Bisetti, F., "Statistics and scaling of turbulence in a spatially developing mixing layer at $\operatorname{Re}_{\lambda}=250, "$ Physics of Fluids, Vol. 24, 2012, pp. 035109-1-035109-21.

${ }^{21}$ Wang, J. K., and Milane, R.E., "Large eddy simulation (2D) of spatially developing mixing layer using vortex-in-cell for flow field and filtered density function for scalar field," International Journal for Numerical Methods in Fluids, Vol. 50, 2006, pp. 27-61.

${ }^{22}$ Zhou X.Y., and Pereira J.C.F., "Large Eddy Simulation (2D) of a Reacting Plane Mixing Layer Using Filtered Density Function Closure," Flow, Turbulence and Combustion, Vol. 64, 2000, pp. 279-300.

${ }^{23}$ McMullan W.A., Gao S., and Coats C.M., "A comparative study of inflow conditions for two- and three-dimensional spatially developing mixing layers using Large Eddy Simulation," International Journal of Numerical Methods in Fluids, Vol. 55, 2006, pp. 589-610.

${ }^{24}$ McMullan W.A., Gao S., and Coats C.M., "Investigation of coherent structures in turbulent mixing layers using Large Eddy Simulation," 48th AIAA Aerospace Science Meeting, Orlando, FL, 2010, AIAA 2010-1291.

${ }^{25}$ Wang Y., Tanahashi M., and Miyauchi T., "Coherent fine scale eddies in turbulence transition of spatially-developing mixing layer," International Journal of Heat and Fluid Flow, Vol. 28, 2007, pp. 1280-1290.

${ }^{26}$ Comte P., Silvestrini J.H., and Bégou P., "Streamwise vortices in Large Eddy Simulations of mixing layers," European Journal of Mechanics B/Fluids, Vol. 4, 1998, pp. 615-637.

${ }^{27}$ McMullan, W.A., and Garrett, S.J., "The Effect of Initial Conditions on Streamwise Vortices in the Plane Turbulent Mixing layer," AIAA Aviation, 2015.

${ }^{28}$ McMullan W.A., Gao, S., and Coats, C.M. "Organised structure in the post-transition mixing layer. Part 2: Large Eddy Simulation," Journal of Fluid Mechanics, Vol. 762, 2015, pp. 302-343.

${ }^{29}$ Nicoud, F., and Ducros, F., "Subgrid-scale stress modelling based on the square of the velocity gradient tensor," Flow, Turbulence and Combustion, Vol. 62, 1999, pp. 183-200.

${ }^{30}$ Xiao, F., Dianat, M., and McGuirk, J.J., "A Recycling/Rescaling Method for LES Inlet Condition Generation," Proceedings of the 8th International ERCOFTAC Symposium on Engineering Turbulence Modelling and Measurements, Marseille, France pp. $510-515$.

${ }^{31}$ McMullan, W.A., and Garrett, S.J., "On the growth of a plane mixing layer from laminar or turbulent initial conditions," AIAA Aviation, Atlanta, GA, 2014, AIAA-2014-3096.

${ }^{32}$ Wang, P. C., and McGuirk, J. J., "Large eddy simulation of high speed nozzle flows - assessment and validation of synthetic turbulence inlet conditions." 20th AIAA Computational Fluid Dynamics Conference, Honolulu, HI, 2011, AIAA-2011-3555.

${ }^{33}$ Xiao, F., Dianat, M., and McGuirk, J. J., "LES of turbulent liquid jet primary breakup in turbulent coaxial flow," International Journal of Multiphase Flow, Vol. 60, 2014, pp. 103-118.

${ }^{34}$ McMullan, W.A., "Spanwise domain effects on the evolution of the plane turbulent mixing layer," International Journal of Computational Fluid Dynamics, to appear.

${ }^{35}$ Bell, J.H., and Mehta, R. D., "Measurements of the streamwise vortical structures in a plane mixing layer," Journal of Fluid Mechanics, Vol. 239, 1992, pp. 213-248.

${ }^{36}$ McMullan, W.A., and Garrett, S.J., "The Effect of Initial Conditions on Streamwise Vortices in the Plane Turbulent Mixing Layer," AIAA Aviation, Dalla, TX, 2015.

${ }^{37}$ Plesniak, M. W., Bell, J. H., and Mehta, R. D., "Effects of small changes in initial conditions on mixing layer threedimensionality,"' Experiments in Fluids, Vol. 14, 1993, pp. 286-288. 


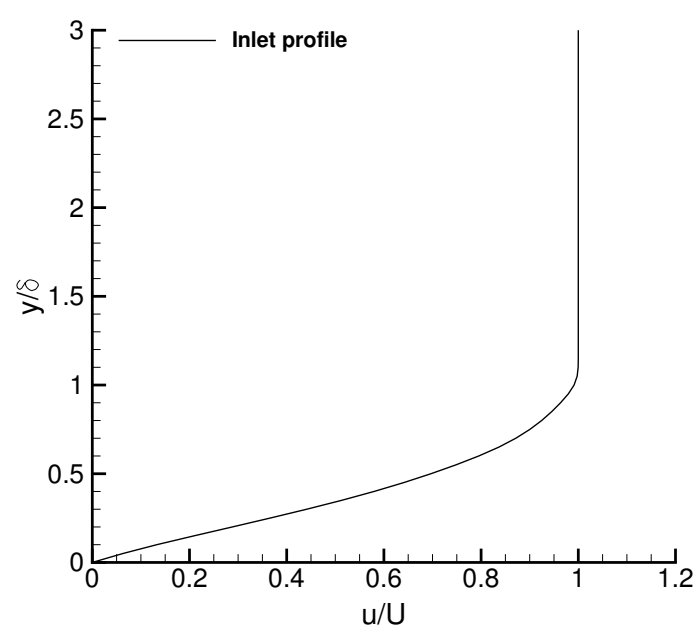

(a) Mean streamwise velocity.

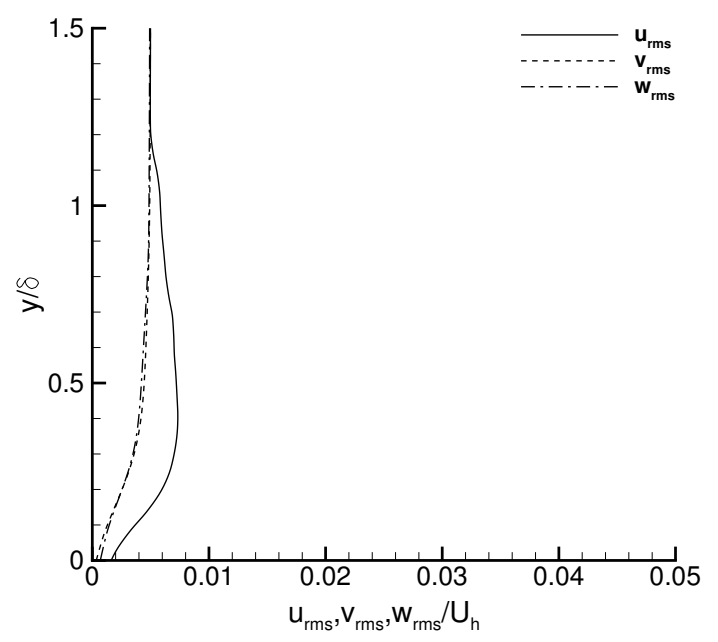

(b) Velocity fluctuation profiles, Cases with suffix 'L'.

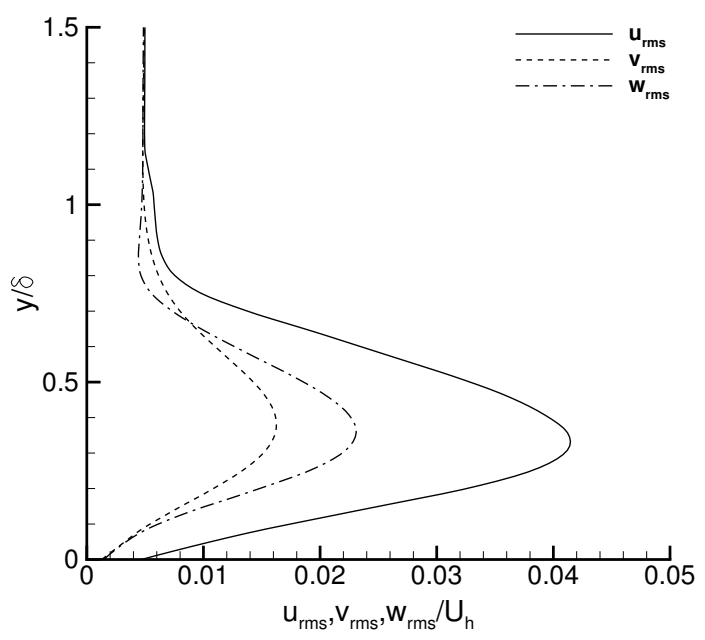

(c) Velocity fluctuation profiles, Cases with suffix ' $\mathrm{H}$ '.

Figure 1. Inflow conditions for the simulations. 


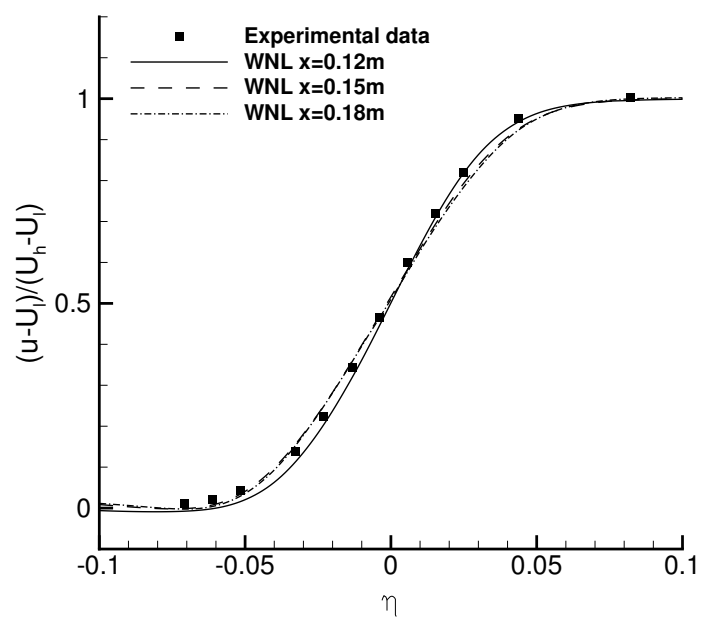

(a) Case WNL.

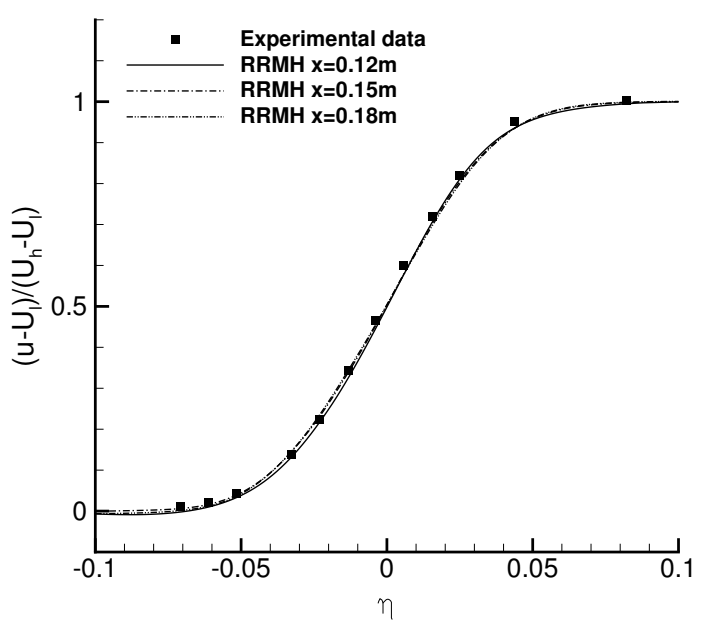

(b) Case RRMH.

Figure 2. Mean streamwise velocity statistics

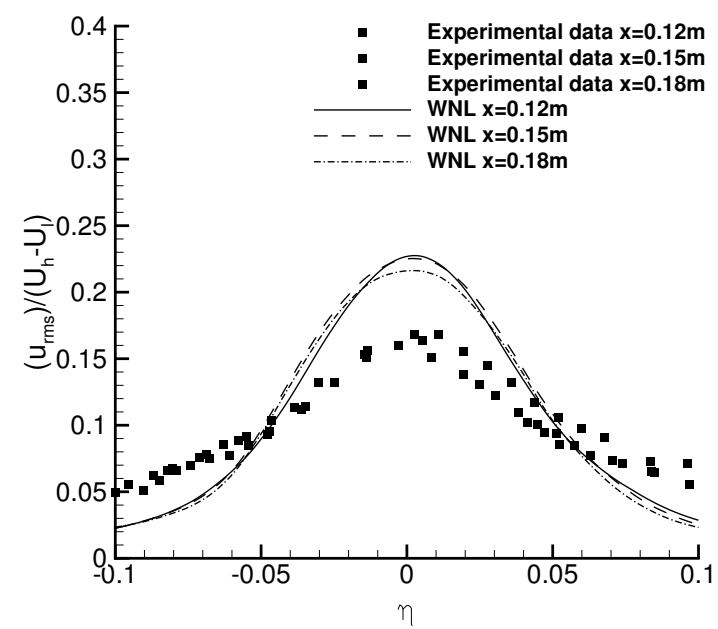

(a) Case WNL.

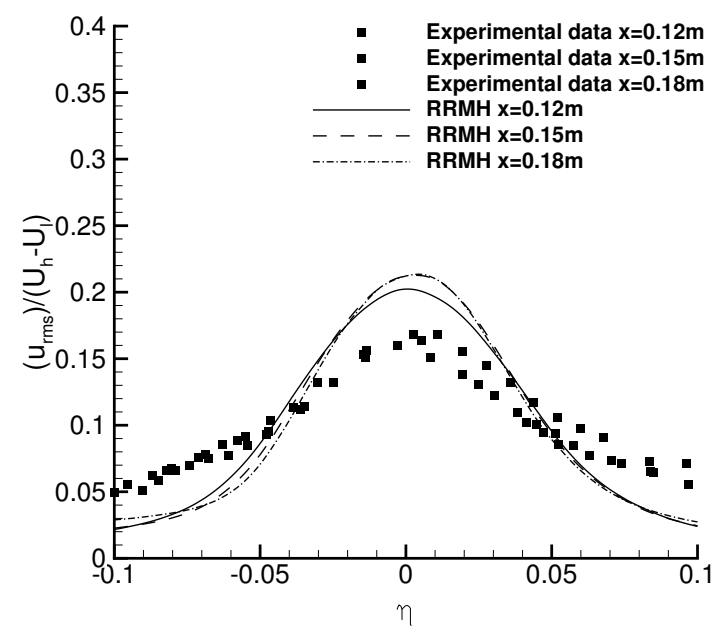

(b) Case RRMH.

Figure 3. Mean streamwise velocity fluctuation statistics

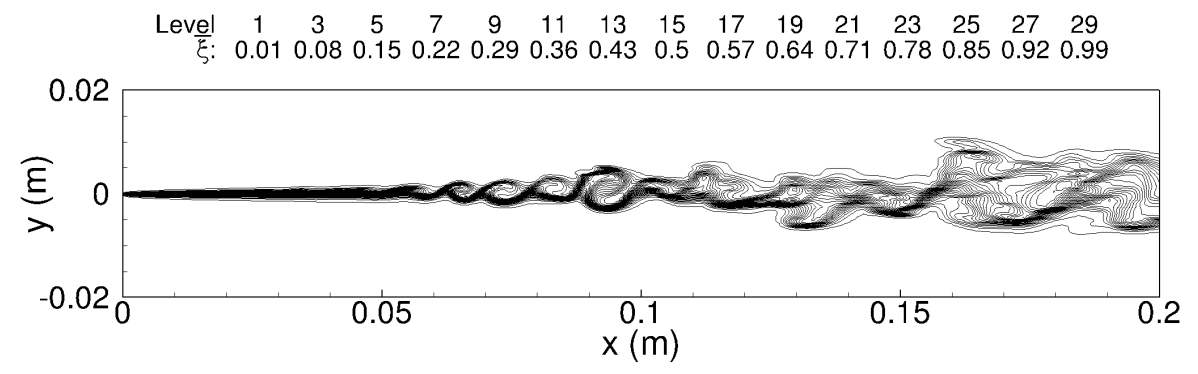

Figure 4. Instantaneous single plane scalar distribution in Case WNL. 


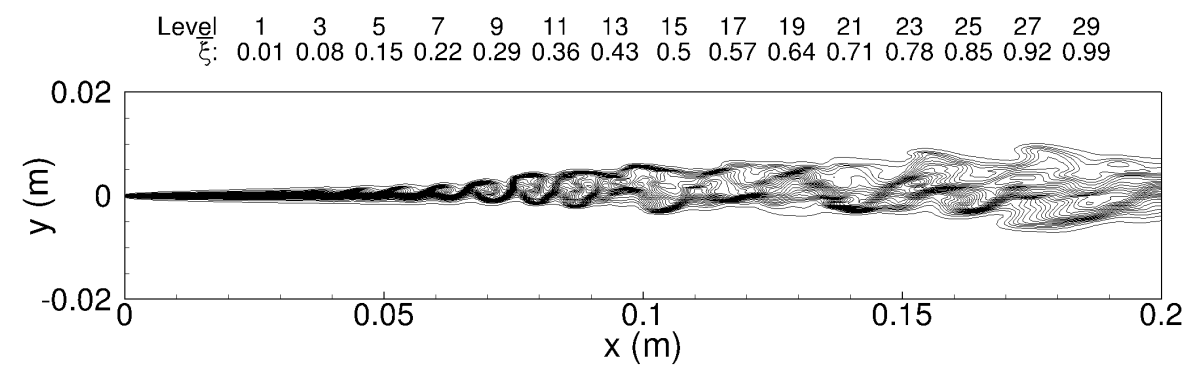

Figure 5. Instantaneous single plane scalar distribution in Case RRML.

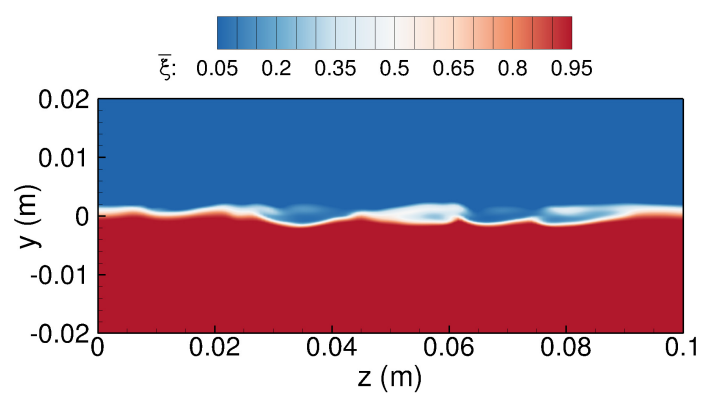

(a) $x=0.07 \mathrm{~m}$

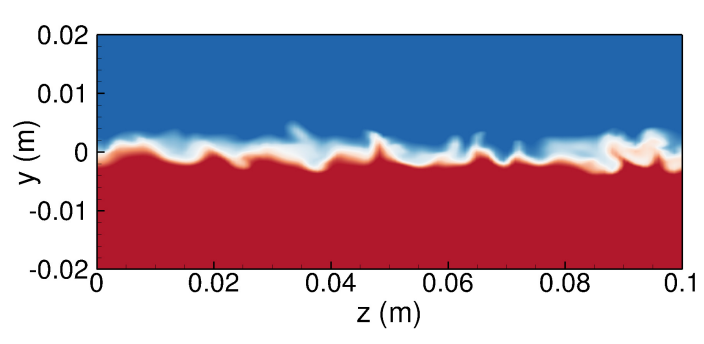

(b) $x=0.11 \mathrm{~m}$.

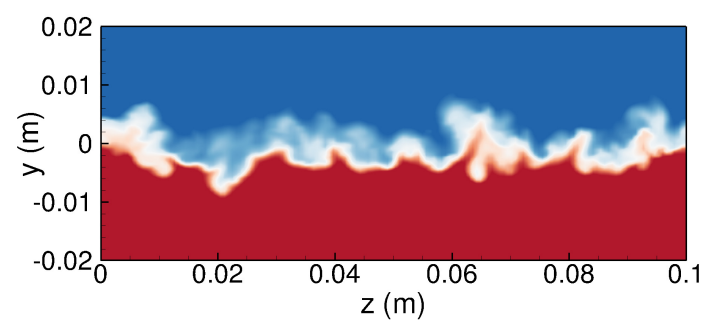

(c) $x=0.15 \mathrm{~m}$.

Figure 6. Instantaneous $y-z$ passive scalar distribution through structure cores in Case WNL. 


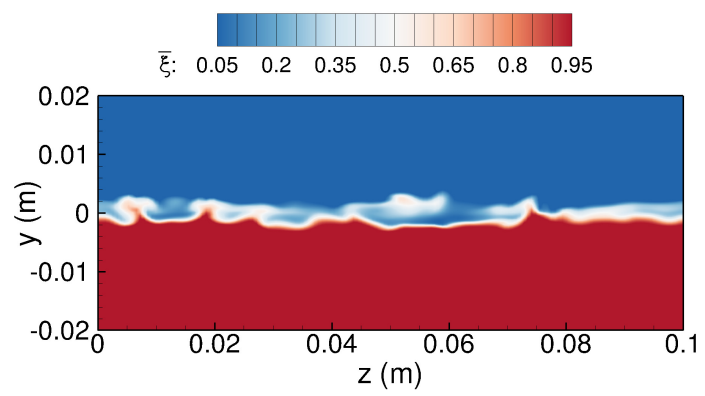

(a) $x=0.07 \mathrm{~m}$.

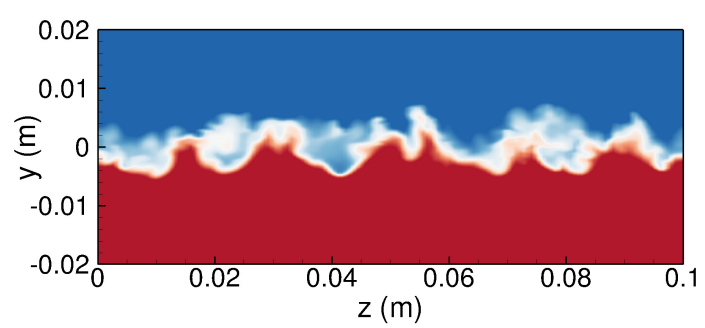

(b) $x=0.11 \mathrm{~m}$.

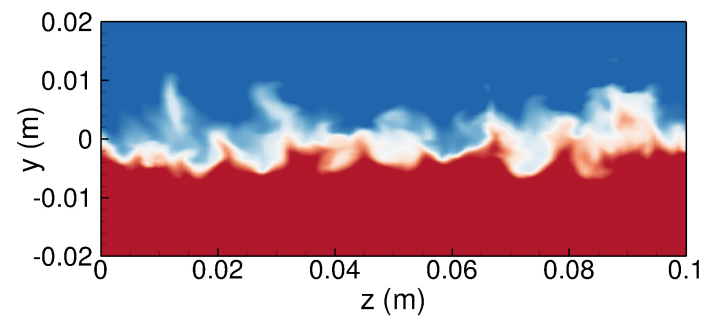

(c) $x=0.15 \mathrm{~m}$.

Figure 7. Instantaneous $y-z$ passive scalar distribution through structure cores in Case WNH.

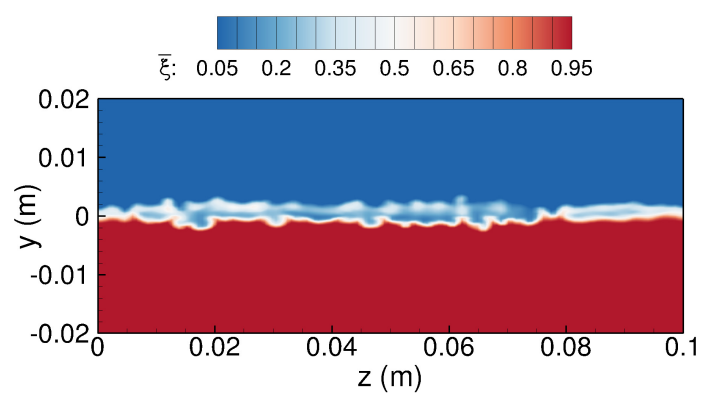

(a) $x=0.07 \mathrm{~m}$.

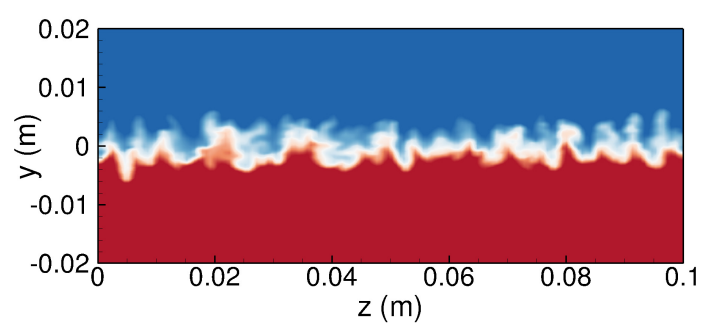

(b) $x=0.11 \mathrm{~m}$.

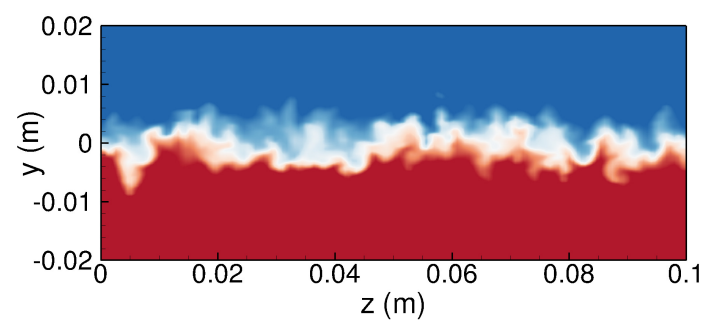

(c) $x=0.15 \mathrm{~m}$.

Figure 8. Instantaneous $y-z$ passive scalar distribution through structure cores in Case RRML. 


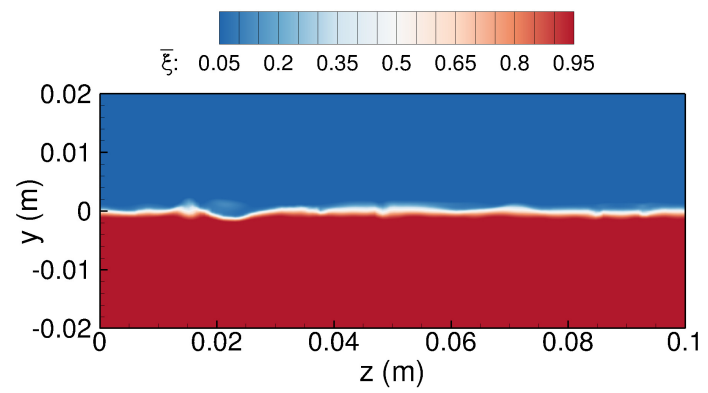

(a) $x=0.07 \mathrm{~m}$.

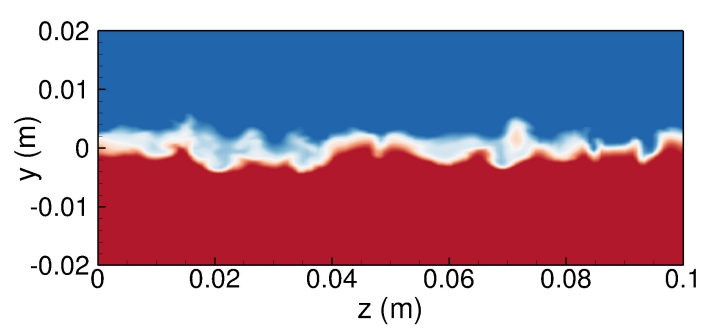

(b) $x=0.11 \mathrm{~m}$.

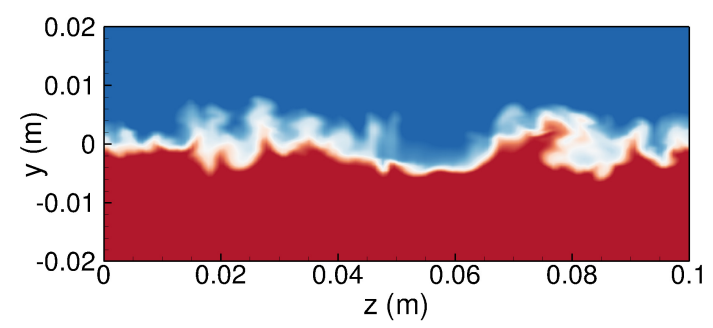

(c) $x=0.15 \mathrm{~m}$.

Figure 9. Instantaneous $y-z$ passive scalar distribution through structure cores in Case RRMH. 


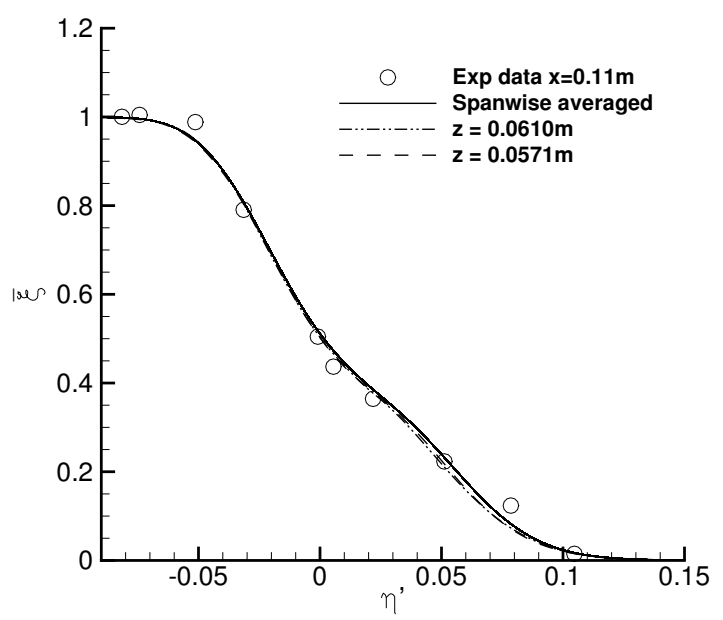

(a) Case WNL.

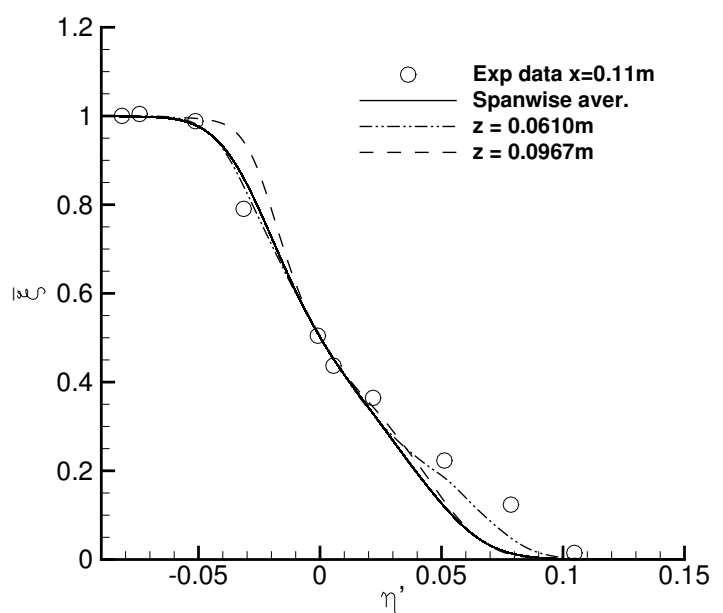

(c) Case RRML.

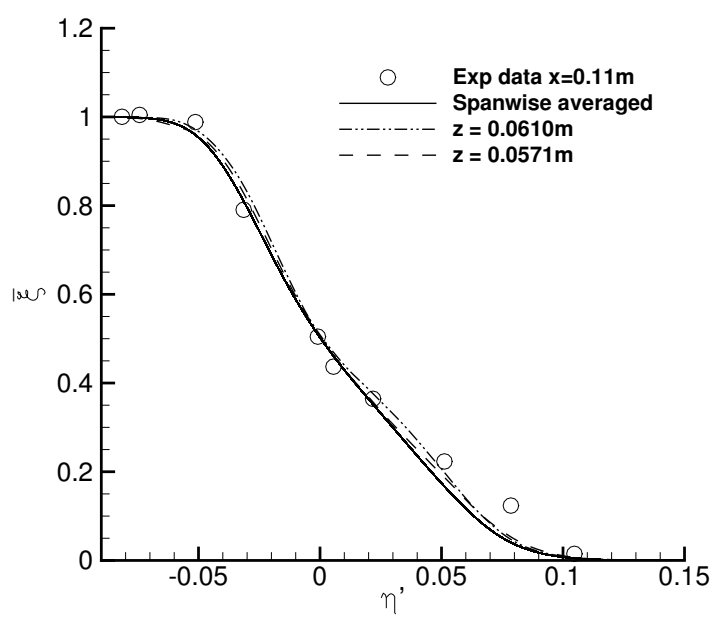

(b) Case WNH.

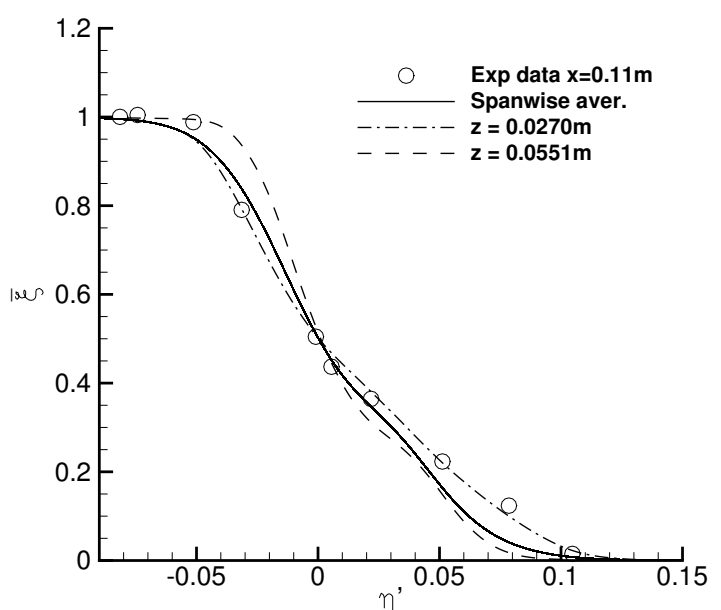

(d) Case RRMH.

Figure 10. Mean scalar profiles in the simulations, recorded at $x=0.11 \mathrm{~m}$. 


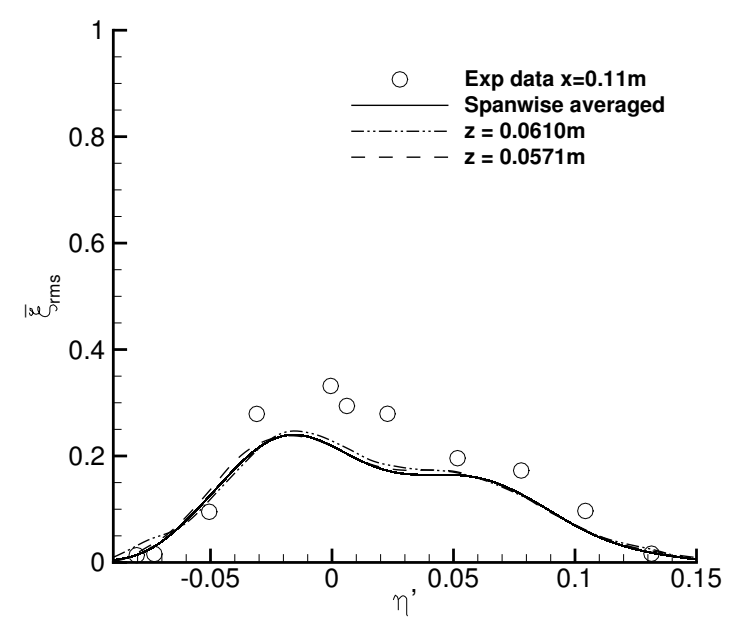

(a) Case WNL.

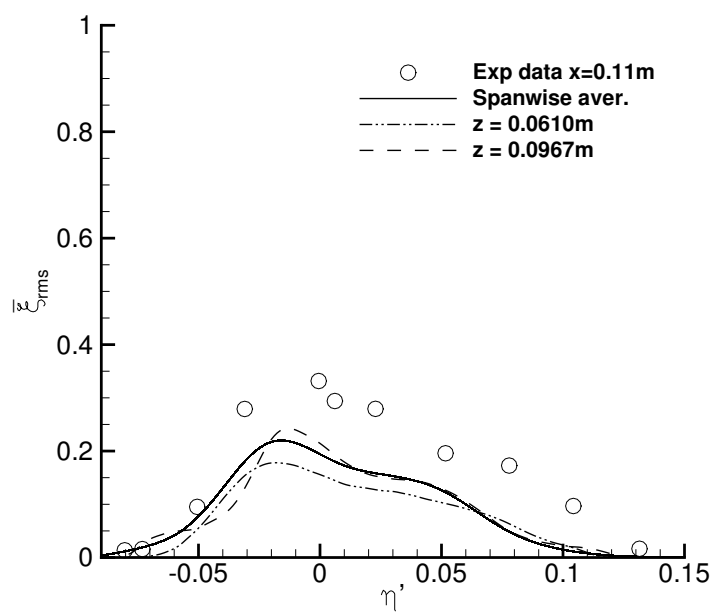

(c) Case RRML.

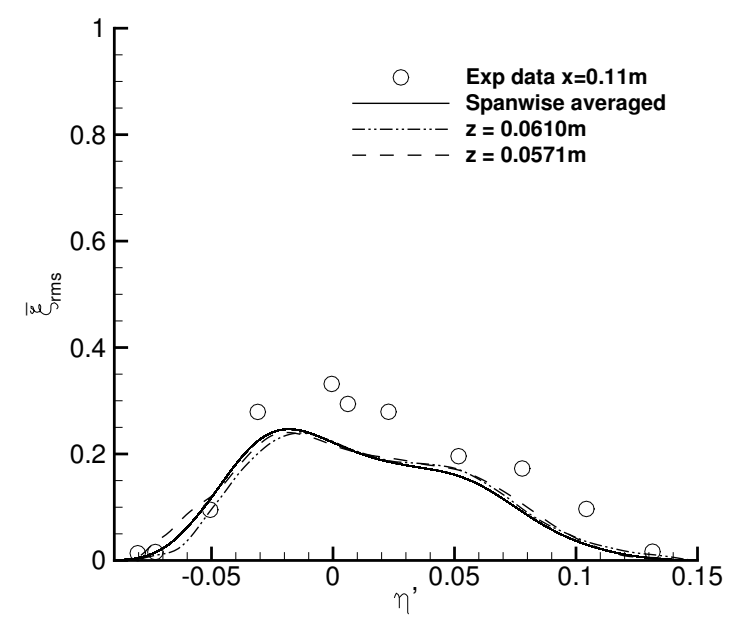

(b) Case WNH.

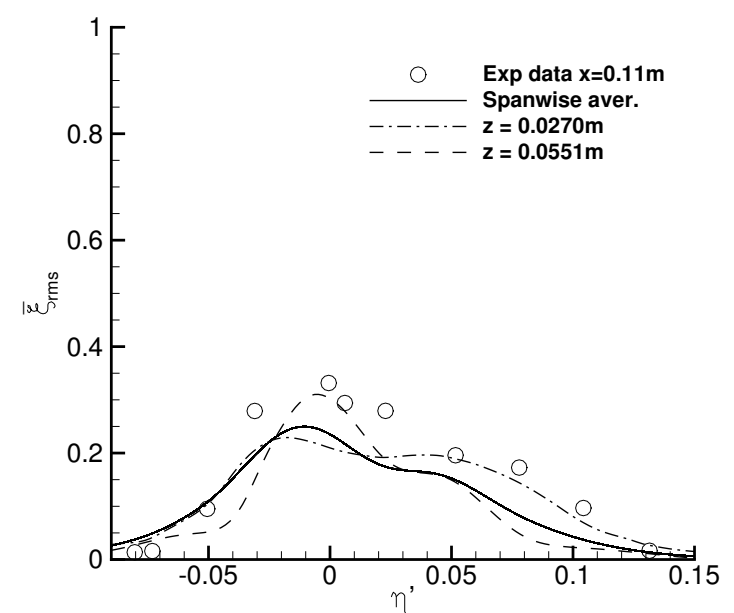

(d) Case RRMH.

Figure 11. Scalar fluctuation profiles in the simulations, recorded at $x=0.11 \mathrm{~m}$. 


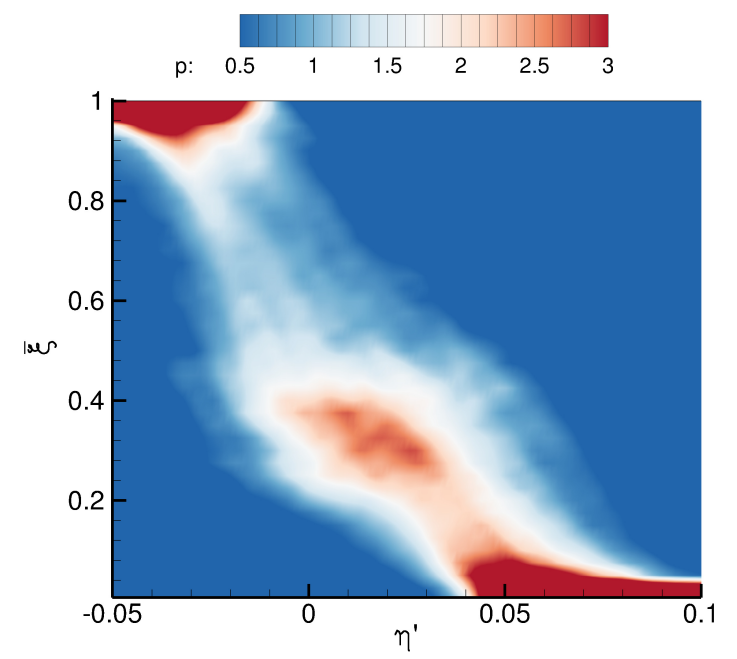

(a) $z=0.0571 \mathrm{~m}$.

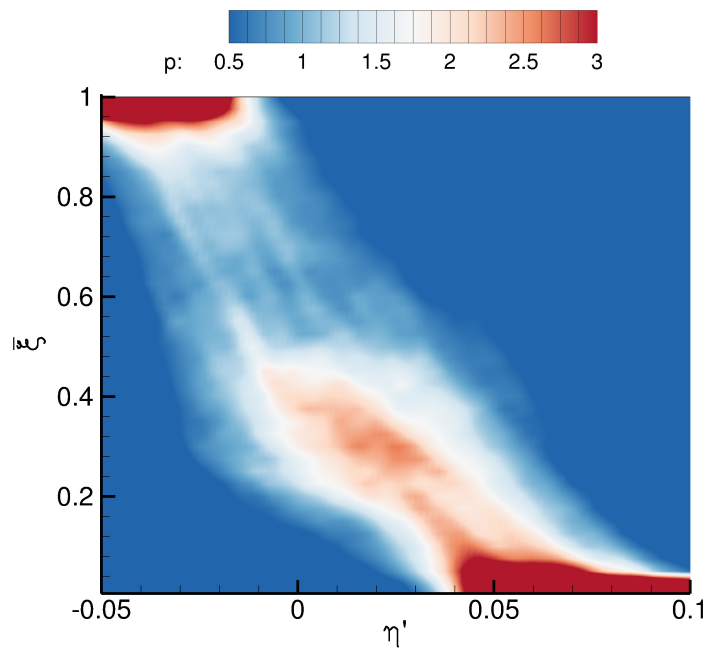

(b) $z=0.0061 \mathrm{~m}$.

Figure 12. Probability Density Functions recorded at $x=0.11 \mathrm{~m}$ in Case WNL.

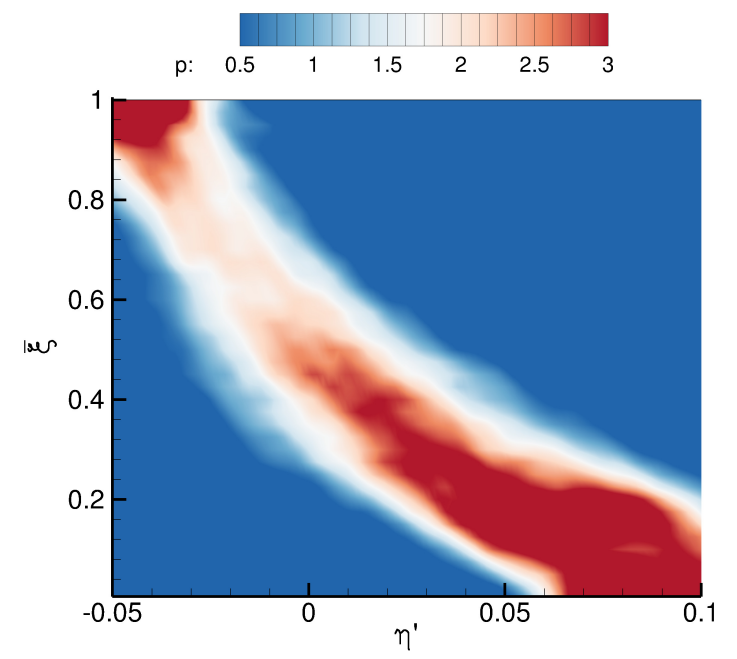

(a) $z=0.061 \mathrm{~m}$.

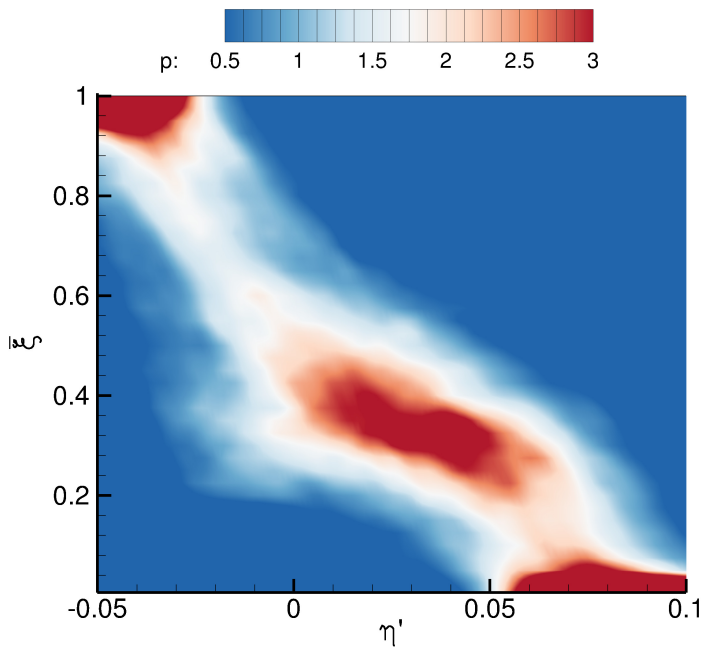

(b) $z=0.0967 \mathrm{~m}$.

Figure 13. Probability Density Functions recorded at $x=0.11 \mathrm{~m}$ in Case RRML. 


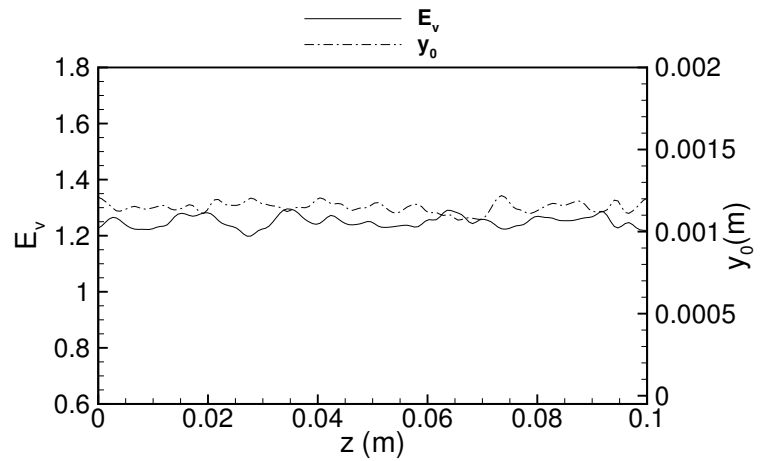

(a) Case WNL.

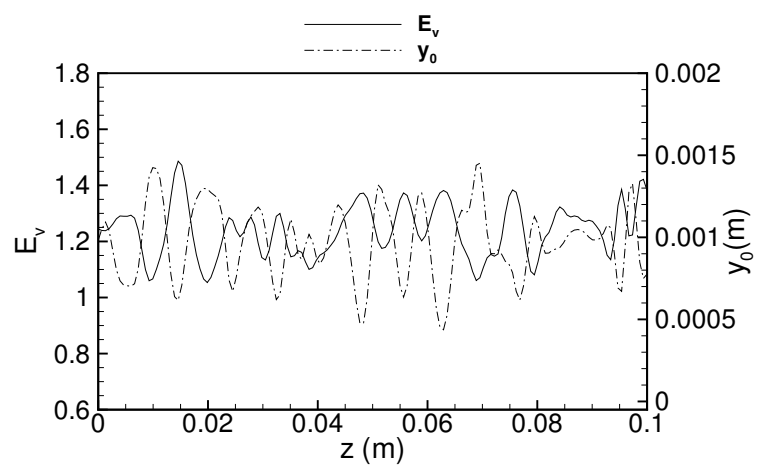

(c) Case RRML.

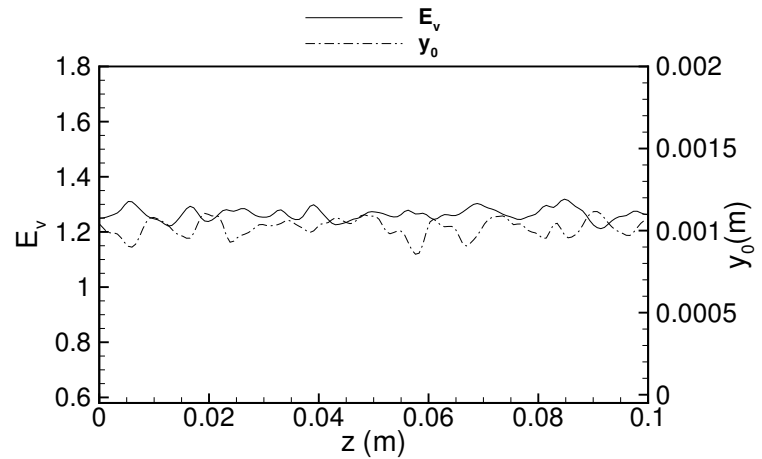

(b) Case WNH.

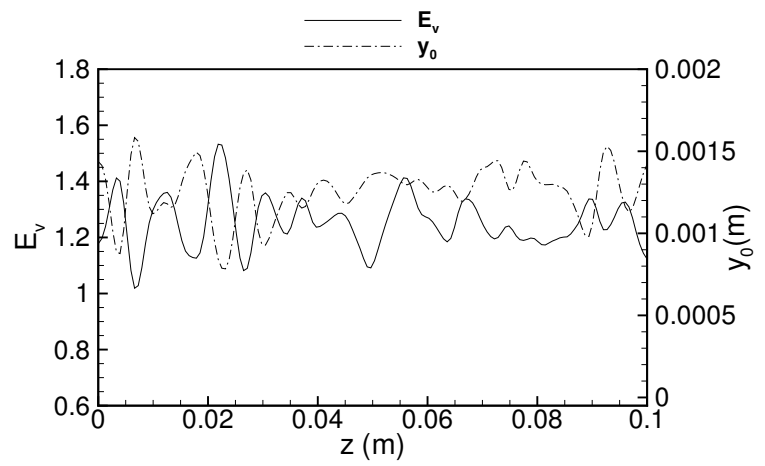

(d) Case RRMH.

Figure 14. Entrainment ratio and mixing layer centreline profiles obtained at $x=0.11 \mathrm{~m}$.

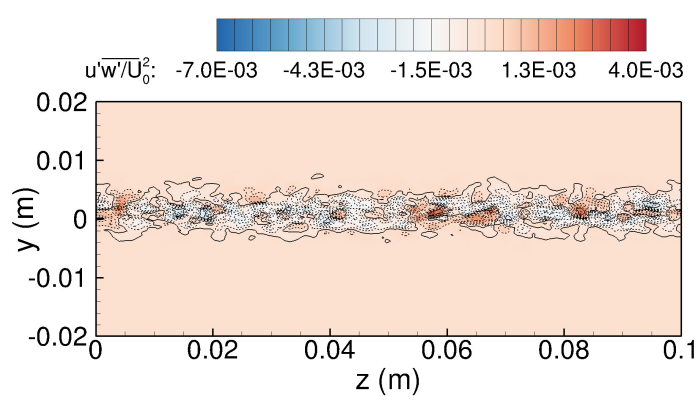

(a) Case WNL.

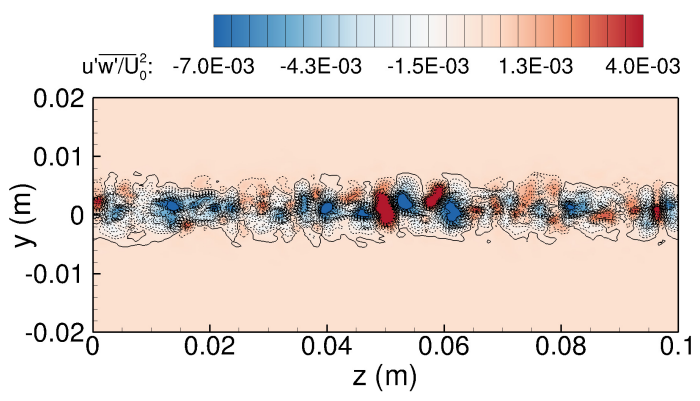

(c) Case RRML.

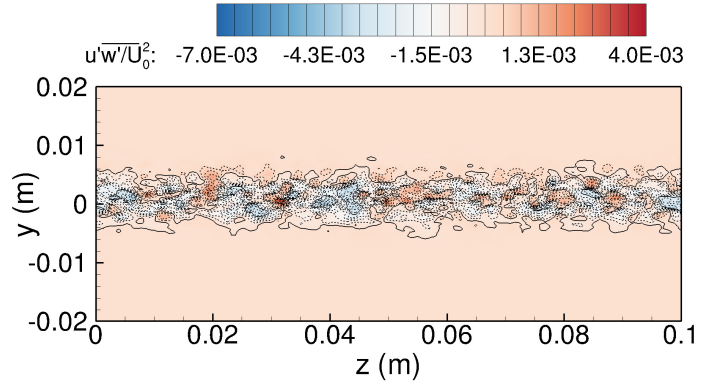

(b) Case WNH.

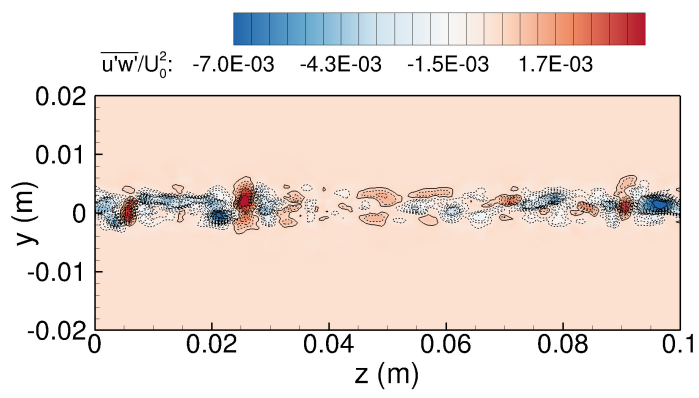

(d) Case RRMH.

Figure 15. Normalised secondary shear stress contours recorded at $x=0.11 \mathrm{~m}$. 